\title{
Research on distance education image correction based on digital image processing technology
}

Ling Ma

\begin{abstract}
Distance education is generally developed through live broadcast or video playback. Because of the influence of various factors in the process of distance education, the pixel characteristics in the original educational resource image will change. Based on this, this study is based on digital image processing technology to correct the distance education image. In addition, this study uses the layered processing model to decompose each color channel and then process the image brightness channel, which effectively reduces the computational overhead while ensuring the fusion effect. Finally, combined with comparative experimental research, the performance analysis of the algorithm is carried out. Research shows that the algorithm of this study has good performance in image correction and can provide theoretical reference for subsequent related research.
\end{abstract}

Keywords: Digital image, Image processing, Remote, Network education, Image correction

\section{Introduction}

In distance education, image resources are an important part of distance learning resources. Image is an important form of conveying teaching information and carrying educational content. In the teaching process, a variety of media information integrating text, graphics/images, audio and even video technologies can stimulate the learner's interest in learning and improve the quality of teaching. In distance education, the greatest advantage of images is their figurativeness. Many of the contents of teaching will appear "dry" and boring if they are only expressed in words, and the expression will become very "rich and colorful" with images. In the so-called a picture wins thousands of words, image teaching is conducive to fully activate the classroom learning atmosphere and inject vitality into teaching [1]. In network distance education, due to various factors, remote network education will be limited by different specifications in the image transmission process, resulting in a large amount of noise in the image, which causes a large amount of noise in the image, causes the pixel features in the original educational resource image to change, makes the

Correspondence: cqmaling2009@163.com

School of Information Engineering, Chongqing Industry Polytechnic College, Chongqing 401120, China

(c) The Author(s). 2019 Open Access This article is distributed under the terms of the Creative Commons Attribution 4.0 International License (http://creativecommons.org/licenses/by/4.0/), which permits unrestricted use, distribution, and reproduction in any medium, provided you give appropriate credit to the original author(s) and the source, provide a link to the Creative Commons license, and indicate if changes were made. color of the distance education resource image visually error, and leads to distortion of the image setting. The above situation will make students' attention divergence, less interest in learning, and lack of positive learning motivation [2]. If the image cannot be set reasonably and the image noise is reduced, the image information transmitted in the distance education will be inaccurate, and the image will lose the meaning of the demonstration, which will make the students unable to understand the deep meaning of the image information description and reduce the teaching effect and quality [3]. Only by filtering the noise signal in the image to ensure that the image storage valuable information is accurately displayed can it play an important role in network distance learning. Therefore, this study uses image processing technology to achieve distance education image correction.

In many applications such as remote sensing, medical imaging, security, computer vision, multi-camera video and panoramic image, correcting nonlinear distortion caused by optical lens has always been a hot research topic [3]. The distortion correction of the image can be generally started from two aspects, optical design correction and digital image processing correction. The use of optical and mechanical structures for lens correction has many limitations in terms of design difficulty, \\ SpringerOpen}


manufacturing, and lens size and cost. Therefore, correction using digital image processing has become the mainstream of society. The use of digital image processing technology to achieve image correction of the camera is usually divided into two steps, camera calibration and image correction [4].

Establishing the geometric model of camera imaging and determining the parameters of the camera model are the main content of camera calibration in computer vision, and it is also the primary task of image correction. The internal and external parameters of the camera determine the mapping relationship between the 3D scene and the corresponding 2D image. The process of restoring and determining the parameters inside and outside the camera is called camera calibration. The internal optical and geometric characteristics of the camera determine the camera's internal parameters, such as camera focal length, distortion factor, and image center. However, the camera's external parameters represent the three-dimensional position and orientation information of the world coordinate system and camera coordinate system [5]. Since the end of the nineteenth century, camera calibration and lens correction have been the research hotspots in academia. The development of this research hotspot has formed a very perfect theoretical basis, and many algorithms have been proposed to improve the calibration results from accuracy and speed [6].

In 1966, B. Hallert first used the least squares method to process the observation data obtained in camera calibration, thus obtaining high-precision results in field measurements [7]. Abdal. Aziz and Karara proposed the direct linear transformation (DLT) camera calibration method in the early 1970s. From the perspective of photogrammetry, the relationship between camera images and 3D world was deeply studied, and a linear model was established. The linear model is easy to calculate and fast, but it is difficult to fully represent the nonlinear distortion characteristics of the camera [8]. In the mid-1980s, R.Tsai proposed a RAC-based calibration method and established a classic Tsai camera model. The core of the method is to solve the linear parameters by using the linear model and then iteratively solve the nonlinear parameters according to the radial uniform constraints. The distortion model based on RAC method reduces the complexity of parameter solving, and the calibration process is fast and accurate [9]. Later, J. Weng improved Tsai's distortion model, which made it compatible with distorted lenses [10].

ZhengYou Zhang proposed a flexible plane calibration method to simplify the process of camera calibration while obtaining high-precision calibration results. The method takes three or more images based on black and white checkerboard templates of different angles and postures, extracts the corner coordinates of the chessboard in the image, and substitutes the established camera model equations to obtain the internal and external parameters and distortion coefficients of the camera. Later, camera calibration methods based on circular templates, hexagonal lattice patterns, etc., were developed [11].

With the development of computer automation technology since the twentieth century, a new type of active vision camera calibration method and camera self-calibration method have been formed. The active visual calibration method based on camera two orthogonal motion proposed by Hu Zhanyi et al. is easier to implement and can solve all five internal parameters of the camera compared with the three orthogonal motion method proposed by Ma Weide. Traditional calibration methods and active visual calibration are inseparable from special scenes or camera-related motion information. In order to meet the needs of camera calibration under the unknown motion of any scene, the self-calibration of the camera is proposed. It can be roughly divided into self-calibration method based on Kruppa equation and self-calibration method based on absolute quadric surface and infinity plane [12].

Through the above analysis, we can see that the current digital image technology is less used in network education image correction. Therefore, this study analyzes the image problems in remote network education and combines the digital image processing technology to analyze the remote network image correction and, on this basis, draws an effective strategy.

\section{Research methods}

When the color in the image is rich and evenly distributed, the gray world method can be used to obtain an ideal correction result. However, for some specific scenes, such as the existence of a large area of a single color in the image, the premise of the gray world method assumption is no longer satisfied, and the corrected image will produce a significant color deviation. The richer the image color, the more accurate the color reproduction during calibration. According to the definition of entropy, image entropy can be used to measure the richness of image color. The richer the image color, the larger the image entropy; conversely, the less the color in the image, the smaller the image entropy. Applying the physical meaning of image entropy to the algorithm, firstly, the image is evenly segmented, the entropy values of different sub-blocks are different, and the weights are assigned according to the sub-block entropy value, thereby reducing the influence of a single-color block. Based on the image entropy theory, the gray world algorithm is improved, and the application range of the algorithm is expanded. First, the histogram 
equalization processing is performed on the R, G, and B channels of the image to improve the overall contrast. After the equalization process, the color cast of the color cast image has been weakened and the image contrast is deepened [13].

When the image is chunked, the choice of partition size is important. Considering the distribution of the pixel level, the image is divided into sub-blocks $T_{i j}(1 \leq i \leq m, 1 \leq$ $j \leq n)$ of $16 \times 16$ size, which are divided into $n \times \mathrm{m}$ image blocks, and the block mode is as shown in Fig. 1. The three-channel image entropy of the image sub-blocks $R$, G, and B is calculated by using Eq. (1), which are denoted as $H_{i j}^{\mathrm{R}}, H_{i j}^{\mathrm{G}}$, and $H_{i j}^{\mathrm{B}}$, and the mean value is taken as the image entropy of the image block [14]:

$$
E_{i j}=\left(H_{i j}^{\mathrm{R}}+H_{i j}^{\mathrm{G}}+H_{i j}^{\mathrm{B}}\right) / 3
$$

The larger the entropy value of the image block and the richer the color of the image block, the more accurate the color reduction during correction, and the image obtained when the gray world method is used for correction is also the expected image. Therefore, in order to reduce the influence of a single-color block on image correction, a color-rich image block is given a higher weight level, and a color-only image block is given a lower weight level. The concept of image entropy is used to measure the color richness of sub-blocks, and weights are assigned according to their size. The larger the entropy value of the image block, the larger the weight of the allocation; conversely, the smaller the entropy value of the image block, the smaller the weight assigned to it. Therefore, after calculating the image entropy of all sub-blocks, the sub-block weight value is calculated by normalizing the image entropy, and the calculation formula is [15]:

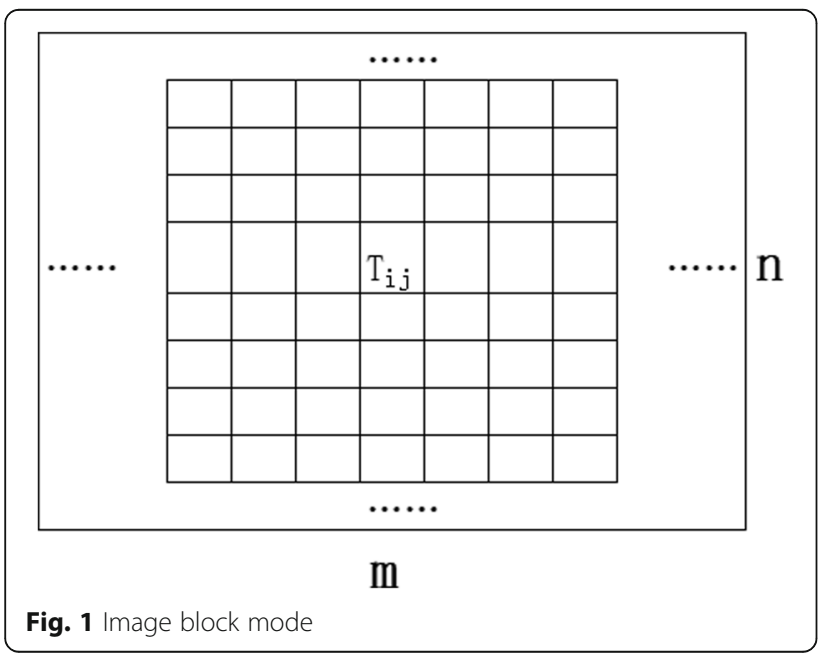

$$
W_{i j}=\frac{E_{i j}}{\sum_{i=1}^{m} \sum_{j=1}^{n} E_{i j}}
$$

Among them, $W_{i j}$ is the corresponding weight of any image block $T_{i j}(1 \leq i \leq m, 1 \leq j \leq n)$, and $E_{i j}$ is the image entropy of the sub-block. The average values of the three channels R, G, and B of each image block are counted and recorded as $\overline{\mathrm{R}_{i j}}, \overline{G_{i j}}$, and $\overline{B_{i j}}$. According to the weight value of the sub-block entropy value, the mean R, G, and B channel values [16] of the whole image are calculated:

$$
\left\{\begin{array}{l}
E W_{-\bar{R}}=\sum_{i=1}^{m} \sum_{j=1}^{n} W_{i j} \times \overline{\mathrm{R}_{i j}} \\
E W_{-\bar{G}}=\sum_{i=1}^{m} \sum_{j=1}^{n} W_{i j} \times \overline{\mathrm{G}_{i j}} \\
E_{-} W_{B}=\sum_{i=1}^{m} \sum_{j=1}^{n} W_{i j} \times \overline{\mathrm{B}}
\end{array}\right.
$$

After obtaining the weighted mean value of the entropy values of the $R, G$, and $B$ channels, the average value of the three-channel entropy is taken as the average gray value of the image, namely:

$$
\text { Gray }=\left(E W_{-\bar{R}}+E W_{-\bar{G}}+E_{-} W_{B}\right) / 3
$$

The Von Kries diagonal model is applied to update the $\mathrm{R}, \mathrm{G}$, and $\mathrm{B}$ components of each pixel in the image, and corrects the entire image, and adjusts the corrected pixels to a displayable range $[0,255]$.

$$
\left\{\begin{array}{l}
R^{\prime}=\left\{\begin{array}{c}
R * R_{\text {Gain }} \rightarrow R * R_{\text {Gain }}<255 \\
255 \rightarrow R * R_{\text {Gain }}>255
\end{array}\right\} \\
R^{\prime}=\left\{\begin{array}{c}
G * G_{\text {Gain }} \rightarrow G * G_{\text {Gain }}<255 \\
255 \rightarrow G * G_{\text {Gain }}>255
\end{array}\right\} \\
R^{\prime}=\left\{\begin{array}{c}
B * B_{\text {Gain }} \rightarrow B * B_{\text {Gain }}<255 \\
255 \rightarrow B * B_{\text {Gain }}>255
\end{array}\right\}
\end{array}\right.
$$

For imaging devices such as cameras, there is a large difference between the colors of images acquired under different lighting conditions. In the normal color temperature environment, the acquired image has no color cast, which is basically consistent with human visual perception. However, when the color temperature is high, the image is blue overall, and when the color temperature is low, the image is yellowish overall. Figure 2 shows the imaging of the same scene at different color temperatures, and it can be seen that there is a significant difference between the two.

When white balance correction is performed on images acquired under different lighting conditions, if the image is always corrected with a uniform coefficient, it is impossible to obtain a better correction result under various conditions. Therefore, before correcting the image, it is necessary to first detect the color cast of the image to determine whether there is color cast. If it is a color cast image, its type needs to be determined. After knowing the specific color cast of the image, the image can be 


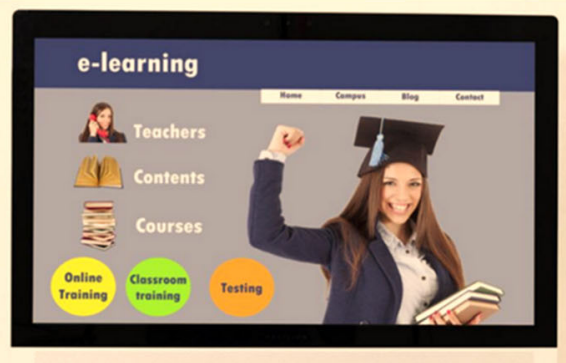

(a) Yellowish image

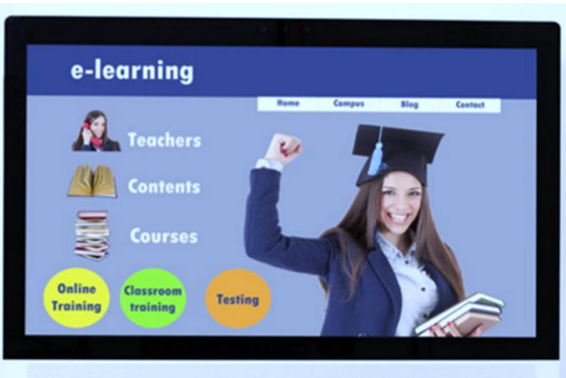

(b) Bluish image

Fig. $\mathbf{2}$ a, b Color cast image at different color temperatures

processed with different coefficients in a targeted manner. According to this idea, an automatic white balance algorithm based on image color cast detection is proposed.

When measuring the degree of deviation between two colors, for the commonly used RGB color space, the difference between the two colors calculated does not correctly represent the true difference between the two colors that people actually perceive. According to the study of color space theory, the CIE Lab color space is close to the perception characteristics of the human visual system, and the distance between the colors calculated by the space is in line with people's actual perception. Therefore, the CIE Lab color space is used to examine the color cast of the image.

The color cast of the image is related to the chromaticity distribution characteristics and the chromaticity mean of the image. When there is a color cast in the image, the two-dimensional histogram distribution on the ab chromaticity coordinate plane is concentrated, which is basically a single peak. At the same time, the larger the chromaticity mean, the larger the color deviation. In contrast, if the two-dimensional histogram distribution is more scattered and there are obvious multi-peaks, the color cast is weakened. Therefore, the concept of equivalent circle is introduced, and the ratio of image mean chromaticity to chromaticity center distance is used to measure the degree of color shift of the image. The color cast factor $K$ is defined as the ratio of the average chromaticity $D$ of the image to the center distance $C$ of the chromaticity. The calculation method is as shown in Eq. (6). The average chromaticity $D$ and the chromatic center distance $C$ are as shown in Eqs. $(7,8)$, respectively.

$$
\begin{aligned}
K & =\frac{D}{C} \\
D & =\sqrt{d_{a}^{2}+d_{b}^{2}}, d_{a}=\frac{\sum_{i=1}^{M} \sum_{j=1}^{N} a}{M N}, d_{b} \\
& =\frac{\sum_{i=1}^{M} \sum_{j=1}^{N} b}{M N}
\end{aligned}
$$

$$
C=\sqrt{C_{a}^{2}+C_{b}^{2}}, C_{a}=\frac{\sum_{a=\text { mina }}^{\max a}\left|a-d_{a}\right|}{M N}, C_{b}=\frac{\sum_{a=\min b}^{\max b}\left|b-d_{b}\right|}{M N} P(b)
$$

Among them, $\mathrm{M}$ and $\mathrm{N}$ are the width and height of the image, respectively, in units of pixels. $\mathrm{P}(\mathrm{a})$ and $\mathrm{P}(\mathrm{b})$ are the corresponding histograms of the positions a and b, respectively. On the ab chromaticity plane, the center coordinate of the equivalent circle is $\left(d_{a}, d_{b}\right)$, the radius is $C$, and the distance from the center of the equivalent circle to the origin of the central axis of the ab chromaticity plane $(a=0, b=0)$ is $D$. Within a certain range of the equivalent circle, the image is considered to have no color shift. If it exceeds the range, it is considered to have a color shift, and the farther the deviation is, the larger the color deviation is. The threshold of the color cast factor is $K_{\text {fold }}$. If $K>K_{\text {fold }}$, the image is considered to have a color cast. Otherwise, it is considered to be colorless. Generally, $K_{\text {fold }}$ is taken as 1.

When the picture has a color cast, the image color cast is judged by the specific position of the equivalent circle on the ab chromaticity plane. The color cast grade is divided into reddish, bluish, greenish, and yellowish. When $d_{a} \geq 0,-d_{a} \leq d_{b} \leq k_{1} d_{a}$, the picture is reddish. When $d_{a}<$ $0, d_{a} \leq d_{b} \leq k_{2} d_{a}$, the picture is greenish. When $d_{b}<0,\left|d_{a}\right|$ $<0,\left|d_{a}\right|<\left|d_{b}\right|$, the picture is bluish. In other cases, the picture is yellowish. The schematic diagram of the color cast is shown in Fig. 3. By evaluating and analyzing multiple images, $k_{1}$ is taken as 2 and $k_{2}$ is -2 .

Through the color cast detection, it has been possible to determine the color cast of the image. Therefore, in the white balance correction, different correction coefficients can be used to correct the image according to the specific color cast. In the correction, the image is first histogram equalized. In the RGB color space, the R, G, and $B$ channels are respectively histogram equalized to obtain the equalized image, and the image color cast is initially weakened. The image is then converted from the RGB color space to the $\mathrm{YCbCr}$ color space, and the data $\left(Y_{\text {Hisp }} C r_{\text {Hisp }}, C b_{\text {Hisp }}\right)$ after the histogram 


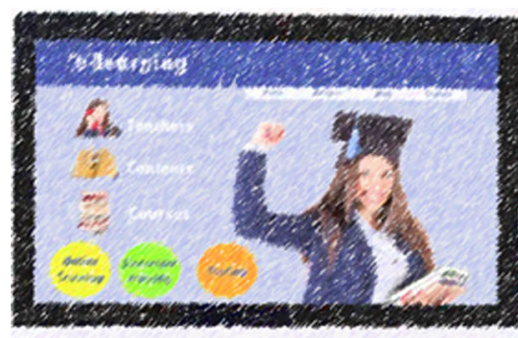

(a) $25 \%$ salt and pepper noise

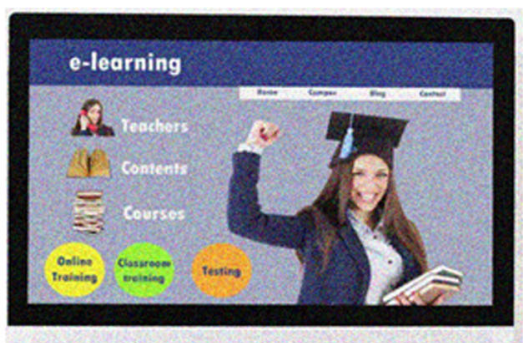

(b) Traditional denoising

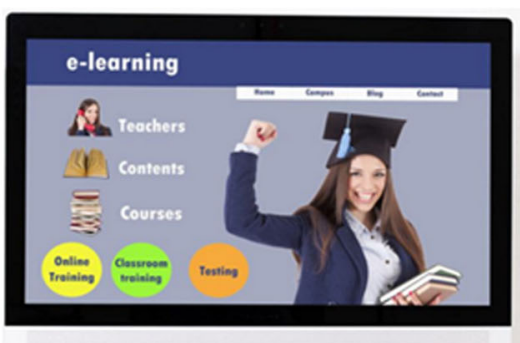

(c) Denoising of the algorithm of this study

Fig. 3 a-c Image denoising results show

equalization of the image is obtained. All pixel points from which Eq. (9) is satisfied are found.

$$
\left\{\begin{array}{c}
Y_{\text {Hist }} \geq 210 \\
-3 \leq C r_{\text {Hisp }}, C b_{\text {Hisp }} \leq+3
\end{array}\right.
$$

In the pixel point satisfying the Formula (9), the highest luminance pixel point $\left(Y_{\text {Hist }}^{\text {bright }}, C r_{\text {Hist }}^{\text {bright }}, C b_{\text {Hist }}^{\text {bright }}\right)$ is found by the maximum value $Y_{\mathrm{Hist}}$ and the value closest to zero $C r_{\text {Hisp }}, C b_{\text {Hisp }}$, and the average value $\left(Y_{\text {Hist }}^{\text {avg }}, C r_{\text {Hist }}^{\text {avg }}, C b_{\text {Hist }}^{\text {avg }}\right)$ satisfying all the pixel points of the Eq. (9) is calculated. Then, all the pixels satisfying the Eq. (10) are found from the histogram-equalized data of the image.

$$
\left\{\begin{array}{c}
Y_{L} \leq Y_{\text {Hist }} \leq Y_{H} \\
C r_{L} \leq C r_{\text {Hist }} \leq C r_{H} \\
C b_{L} \leq C b_{\text {Hist }} \leq C b_{H}
\end{array}\right.
$$

Among them, $Y_{L}$ and $Y_{H} \mathrm{Y}$ are respectively the minimum and maximum values selected between $Y_{H}$ and $Y_{\text {Hist }}^{\text {avg }}, C r_{L}$ and $C r_{H}$ are respectively the minimum and maximum values selected between $C r_{\text {Hist }}^{\text {bright }}$ and $\mathrm{Cr}_{\text {Hist }}^{\text {avg }}$, and $C b_{L}$ and $C b_{H}$ are the same.

For pure white points $(255,255,255)$, the corresponding $\mathrm{YCbCr}$ value is $(255,0,0)$. Therefore, the larger the $\mathrm{Y}$ value, the closer the $\mathrm{CbCr}$ value is to the pixel point of 0 , that is, the closer it is to the white point. The white point in the image is initially selected by the conditional limitation of Eq. (9). Equation (10) further severely selects the conditions for the white point and removes some of the pixel points from the white point just selected, and the remaining pixels are the white spots that satisfy the condition. From the equalized image data $\left(Y_{\text {Hist }}, C r_{\text {Hist }}, C b_{\text {Hist }}\right)$, the pixel points satisfying the corresponding position of the formula (3.14) are selected as the reference white pixels, and the average value $\left(R_{w}\right.$, $G_{w}, B_{w}$ ) of the reference white pixels is calculated.

The color cast is judged on the image. If it is considered that there is no color shift, no processing is performed on the image; if it is judged that there is color shift, the corresponding scale factor is obtained according to the color cast level. The Von Kries transformation is also applied to correct the original data of the image, and the R, G, and B components of the image are updated. So far, the automatic white balance algorithm based on image color cast detection is completed.

$$
\begin{aligned}
R^{\prime} & =\left\{\begin{array}{c}
R * R_{\text {Gain }} \rightarrow * R_{\text {Gain }}<255 \\
255 \rightarrow R * R_{\text {Gain }}>255
\end{array}\right\} \\
G^{\prime} & =\left\{\begin{array}{c}
G * R_{\text {Gain }} \rightarrow * G_{\text {Gain }}<255 \\
255 \rightarrow G * G_{\text {Gain }}>255
\end{array}\right\} \\
B^{\prime} & =\left\{\begin{array}{c}
B * B_{\text {Gain }} \rightarrow B * B B_{\text {Gain }}<255 \\
255 \rightarrow B * B_{\text {Gain }}>255
\end{array}\right\}
\end{aligned}
$$

This study chooses the median filter method for image denoising. The previous median filter is a commonly used arrangement and summary method, which can effectively filter impulse noise and has high application value. However, in the process of system application, the details of the courseware image will be blurred, and the density of the pulse noise will increase. The filtering performance of this method shows a significant downward trend, which seriously hinders the application of multimedia systems in distance education. Therefore, we propose an improved median filtering method to further optimize the original algorithm to ensure the integrity of the course image details, and to solve the contradiction between the traditional algorithm cannot effectively deal with the filtering performance and image detail integrity. The improved median filtering algorithm can not only filter the noise factors in the courseware image, but also ensure the integrity of the courseware image details, which is the first choice for the system.

The past median filter operates on the pixel points of the image by the same operation method, which causes the value of the real signal point to change, and the image has a fuzzy problem, so it is not suitable for the system application of this paper. If the true pixel signal 
point and the signal point contaminated by noise are accurately distinguished, the misoperation of the true pixel signal point can be avoided, and the clarity of the courseware image can be improved. Using the improved switch median filtering method, the pixel of the courseware image can be accurately judged to avoid misoperation of the true pixel signal point. For a pixel of a suspected color image, there is a strong correlation between adjacent points, and the brightness value of a point in the image is close to the brightness value of the adjacent point. The pixel points of the isolated point of the pixel and the image portion of the edge portion are generally considered to be noise points. Therefore, in a courseware image, if there is a large difference between the value of a certain pixel point and its neighborhood value, the correlation between the pixel point and the neighborhood is low, and the point is considered to be a noise point, which needs to be performed filter processing. If the point value is close to the neighbor value, it is the true signal point.

The extreme median filtering algorithm evaluates noise, which has a strong processing power for large impulse noise. However, some thin lines in the color courseware image and the narrower edge details are also significantly different from the pixel values of the adjacent areas. At this time, the extreme median filtering algorithm will misjudge such pixel points into noise points, and accurate filtering of image noise points cannot be achieved.

Since the traditional median filtering algorithm has certain limitations, it needs to be optimized. Therefore, an improved median filtering algorithm based on median filtering theory is proposed. The improved method uses a method of summarizing between partitions to take a pixel point to be analyzed as a center point $((2 N+$ $1) \times(2 N+1)$ ) square range. The range is set as a window, and pixels in the window range can be arranged according to the brightness level, and the arranged pixel values are divided into $2 N+1$ small ranges. The mean of $2 N+1$ pixel values in the middle small range is used to describe the new pixel brightness at the center point. Unlike the conventional method, this method reduces and eliminates the high-frequency components of the Fourier region, and the high-frequency components are different from the pixel values of the luminance of the image edges. Therefore, the improved filtering algorithm can remove high frequency components in the image and ensure the flatness of the image. In the process of window image position transformation, the improved median filtering algorithm can maximize the smoothness of the image and improve the integrity of the image details. The specific process is as follows:

(1) In the courseware image, the $(2 N+1) \times(2 N+1)$ filter window is set to fuse the center of the filter window and a pixel in the image center. (2) The brightness values of different pixels in the filtering window are collected. (3) The ascending order is used to sort the luminance values and divide them into $2 N+1$ number of segments, ensuring that each cell contains $2 N+1$ number of pixel luminance values. (4) $2 N+1$ number of pixel luminance values are collected between the central cells, and then, the average value of the pixel luminance values is obtained. (5) The obtained mean value is used to update the pixel value of the corresponding window center area. (6) Processes (3)-(5) are cycled until the corresponding analysis and operation are completed for all pixels. The output pixel of the improved median filtering algorithm has a strong correlation with the $2 N+1$ number of pixel brightness value mean of the central segment of the adjacent range image. According to the correlation between the data points in the window, the damage data can be corrected, the noise points are filtered, the sharpness of the image is improved, and the integrity of the image details is ensured.

\section{Results}

In order to verify the effectiveness of the image correction algorithm in this study, we investigated the image correction effects of Gaussian image processing, neural network image correction, and this algorithm through experimental research. In the actual research, the distance education video picture collection is taken as the research object, and the original image is shown in Fig. 4.

First, the image gradation processing results are compared, and the results obtained are shown in Fig. 5. Among them, Fig. $5 \mathrm{a}$ is a processing result of a Gaussian image, Fig. $5 \mathrm{~b}$ is a processing result of a gamma of a god network image, and Fig. $5 \mathrm{c}$ is a processing result of the processing method of the present study.

Edge recognition is performed on the image, and the results obtained are shown in Fig. 6. Among them, Fig. 6a is a processing result of a Gaussian image, Fig. 6b is a processing result of a gamma of a god network image,

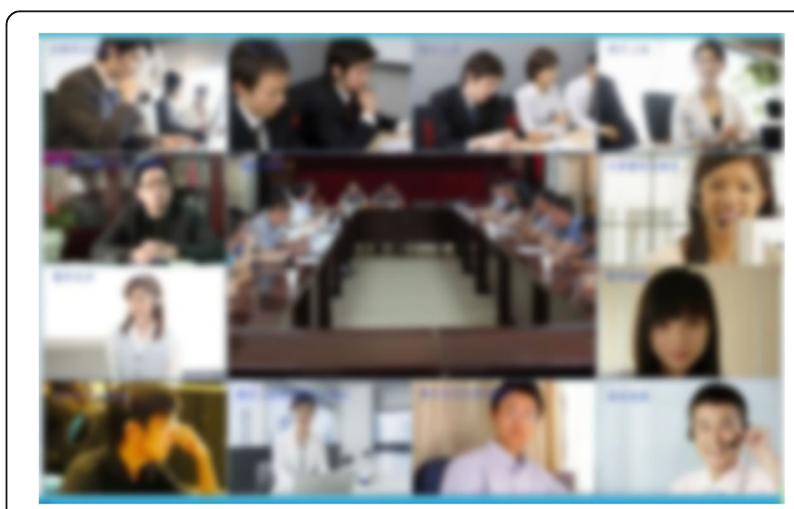

Fig. 4 Original image 


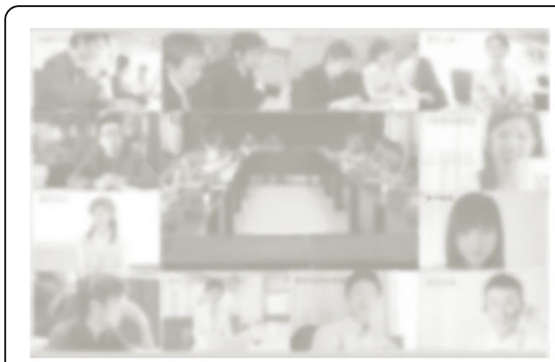

(a)

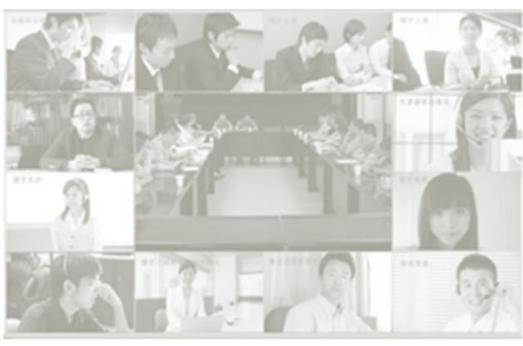

(b)

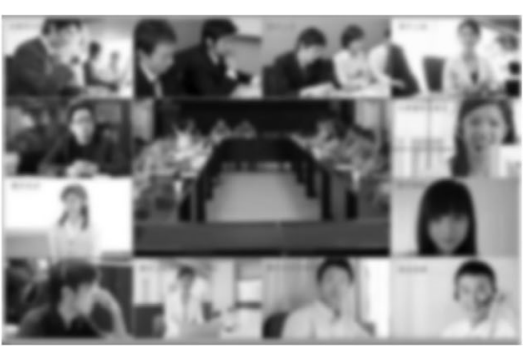

(c)

Fig. 5 a-c Comparison of image grayscale processing results

and Fig. $6 \mathrm{c}$ is a processing result of the processing method of the present study.

The correction effect of the image is compared, and the obtained result is shown in Fig. 7. Among them, Fig. $7 \mathrm{a}$ is a processing result of a Gaussian image, Fig. $7 \mathrm{~b}$ is a processing result of a gamma of a god network image, and Fig. 7c is a processing result of the processing method of the present study.

\section{Discussion and analysis}

The Gaussian image algorithm effectively combines the details of different levels through the multi-resolution decomposition method, so it has a good expressive power for the contrast and saturation of the image, and its color performance is closer to the actual scene. However, the algorithm is not particularly complete for details that are particularly bright or dim, such as the lack of sharpness in Fig. 5a and the clarity of Fig. 7a after image correction. At the same time, due to the pyramid decomposition, its computational overhead is relatively large.

The algorithm of the neural network is simple and easy to implement. For the image with small dynamic range change in Fig. 4, the overall processing effect is not bad. However, when the dynamic range of the image changes greatly, the composite image has obvious color degradation, and the color transition is unnatural. For example, there is a defect in the gradation processing effect shown in Fig. 5b, a relatively significant noise interference in the Fig. $6 \mathrm{~b}$ recognition, and an image enhancement in Fig. 7b, and there are still some unclarities. The color degradation of the image of the method is obvious, and the local boundary is clearly visible, so that the image has a visual feeling similar to that of the oil painting, and at this time, it deviates from the actual perception of the human eye. At the same time, its effect on detail processing is also unsatisfactory, and the overall image feels rather vague and unclear.

The improved algorithm of this research uses the layered processing model to decompose the color channels and then process the image brightness channel. The method effectively reduces the computational overhead while ensuring the fusion effect and avoids the color migration phenomenon caused by ignoring the intrinsic relationship between the color channels in the RGB space. Comparing the correction results of each algorithm, it can be seen that the synthesized image effect is significantly better than the Gaussian algorithm result and the neural network processing result. By observing the gradation processing effect of Fig. $5 \mathrm{c}$, the edge recognition result of Fig. 6c, and the image correction of Fig. 7c, it can be seen that the improved algorithm is very clear on the details, and the subtle parts of each area of different brightness are better expressed. At the same time, the resulting images are more in line with the actual perception of the human eye and are more similar to the real

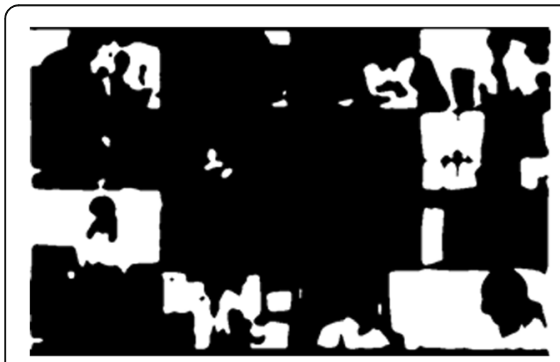

(a)

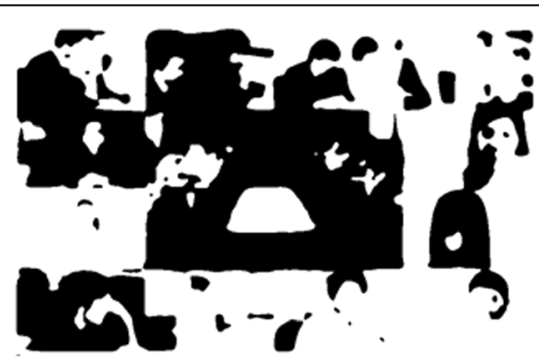

(b)

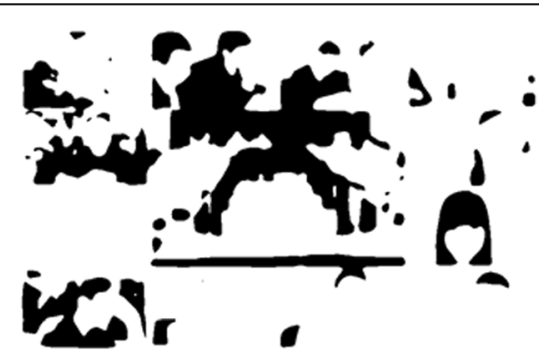

(c)

Fig. 6 a-c Comparison of image edge recognition results 




(a) (b)

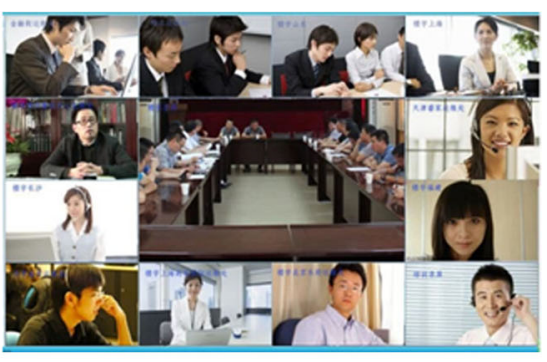

(c)

Fig. 7 Comparison of image correction results

scene. The results were examined using an improved image quality assessment method by referring to Section Results. The image saturation $S$, the information entropy $\mathrm{H}$ and the standard deviation SD are calculated in the HSI color space, and the comprehensive evaluation factor based on the three factors is used as the final evaluation index, and the result image output by each algorithm is analyzed. Overall, the information entropy and standard deviation of the Mertens algorithm are relatively large, that is, the image content and dynamic range expansion are excellent, and the details and definition are complete. Gaussian image processing results have a relatively large saturation, which is an advantage in image color processing, but the processing of detail and definition is slightly inferior. The overall performance of the neural network is average, but its advantage is that it is easy to calculate.

In summary, the improved algorithm yielded better output. Firstly, compared with Gaussian image processing algorithm, the algorithm improves the results of each evaluation factor and effectively draws on the low complexity processing idea of the algorithm. It has advantages in detail content extraction and dynamic range expansion, which is obviously superior to other algorithms. When using the comprehensive evaluation factor to examine the image quality, the research shows that the output of the improved algorithm is significantly better than the Gaussian image processing and neural network image processing, and the results in many aspects are slightly better. Although the improvement is not big with respect to the neural network, the improved algorithm achieves better fusion results while spending less computational overhead, and effectively expands the dynamic range of the image.

\section{Conclusion}

This study analyzes the image problems in remote network education and analyzes the remote network image correction with digital image processing technology. Based on this, an effective strategy is obtained. The study uses image entropy to measure the richness of image color. Firstly, the image is evenly divided. Since there are differences in the entropy values of different sub-blocks, the weights are assigned according to the sub-block entropy values, thereby reducing the influence of a single-color block. For imaging devices such as cameras, there is a large difference between the colors of images acquired under different lighting conditions. In the normal color temperature environment, the acquired image has no color cast, which is basically consistent with human visual perception. The color cast is judged on the image. If it is considered that there is no color shift, no processing is performed on the image; if it is judged that there is color shift, the corresponding scale factor is obtained according to the color cast level. The research proposes an improved median filtering method, which further optimizes the original algorithm and ensures the integrity of the courseware image details, and solves the contradiction between the traditional algorithm and the image detail integrity. Finally, the effectiveness of the proposed algorithm is verified by experiments. It is compared with Gaussian image processing algorithm and neural network image processing algorithm, and the performance superiority of the proposed algorithm is obtained.

\section{Acknowledgements \\ The authors thank the editor and anonymous reviewers for their helpful comments and valuable suggestions.}

\section{Funding}

Not applicable.

\section{Availability of data and materials}

Please contact author for data requests.

\section{Author's contributions}

All authors take part in the discussion of the work described in this paper. The author read and approved the final manuscript.

Competing interests

The author declares that she has no competing interests.

\section{Publisher's Note}

Springer Nature remains neutral with regard to jurisdictional claims in published maps and institutional affiliations. 
Received: 27 October 2018 Accepted: 7 January 2019

Published online: 22 January 2019

References

1. Y. Xu, J.T. Dong, Z.Q. Wang, Fuzzy based distance correction algorithm for digital image interpolation. Appl. Mech. Mater. 513-517(513-517), 15491554 (2014)

2. G. Cao, C.Y. Zhang, Y. Zhang, The traffic accident scene drawing system based on image trapezoid distortion correction. Appl. Mech. Mater. 713715, 1996-1999 (2015)

3. Y. Zhu, B. Chen, M. Qin, et al., 2-D micromachined thermal wind sensors-a review. IEEE Internet Things J. 1(3), 216-232 (2017)

4. S.W. Zhang, X.N. Zhang, Z.Y. Wu, et al., Research on asphalt mixture injury digital image based on enhancement and segmentation processing technology. Appl. Mech. Mater. 470, 832-837 (2014)

5. I. Urdapilleta, L. Dany, J. Boussoco, et al., Culinary choices: a sociopsychological perspective based on the concept of distance to the object. Food Qual. Prefer. 48, 50-58 (2016)

6. V.N. Kopenkov, On halting the process of hierarchical regression construction when implementing computational procedures for local image processing. Pattern Recognit. Image Anal. 24(4), 506-510 (2014)

7. M.K. Hoon, F.A. Cannone, J.J. Hoon, Microstructural analysis of asphalt mixtures using digital image proce. Can. J. Civ. Eng. 41(1), 74-86 (2014)

8. W. Li, X. Zhang, Z. Wang, Music content authentication based on beat segmentation and fuzzy classification. Eurasip J. Audio Speech Music Proc. 2013(1), 11 (2013)

9. S. Vanonckelen, V. Rompaey, et al., The effect of atmospheric and topographic correction methods on land; cover classification accuracy. Int. J. Appl. Earth Obs. Geoinf. 24(1), 9-21 (2013)

10. V. López, A. Fernández, M.J. del Jesus, et al., A hierarchical genetic fuzzy system based on genetic programming for addressing classification with highly imbalanced and borderline data-sets. Knowl.-Based Syst. 38(2), 85104 (2013)

11. G. Bernardini, E. Quagliarini, M. D'Orazio, Towards creating a combined database for earthquake pedestrians' evacuation models. Saf. Sci. 82, 77-94 (2016)

12. K. Adikaram, M.A. Hussein, M. Effenberger, et al., Outlier detection method in linear regression based on sum of arithmetic progression. Sci. World J. 2014, 821623-821623 (2014)

13. M.L. Firdaus, F. Trinoveldi, I. Rahayu, et al., Determination of chromium and iron using digital image-based colorimetry. Procedia Environ. Sci. 20, 298304 (2014)

14. Y. Imamverdiyev, A.B.J. Teoh, J. Kim, Biometric cryptosystem based on discretized fingerprint texture descriptors. Expert Syst. Appl. 40(5), 1888 1901 (2013)

15. J. Poignant, L. Besacier, G. Quénot, Unsupervised speaker identification in TV broadcast based on written names. IEEE/ACM Trans. Audio Speech Lang. Proc. 23(1), 57-68 (2015)

16. J.B. Liu, X.H. Zhang, H.B. Liu, et al., Correction method for non-landing measuring of vehicle-mounted theodolite based on static datum conversion. SCIENCE CHINA Technol. Sci. 56(9), 2268-2277 (2013)

\section{Submit your manuscript to a SpringerOpen ${ }^{\circ}$ journal and benefit from:}

- Convenient online submission

- Rigorous peer review

- Open access: articles freely available online

- High visibility within the field

- Retaining the copyright to your article

Submit your next manuscript at $\boldsymbol{\nabla}$ springeropen.com 